\title{
WPA2 Security-Bandwith Trade-off in 802.11n Peer- Peer WLAN for IPv4 and IPv6 Using Windows XP and Windows 7 Operating Systems
}

\author{
Samad S. Kolahi, Peng Li, Mustafa Safdari, and Mulugeta Argawe
}

\author{
Unitec New Zealand, New Zealand
}

In this paper, we present new results on the performance of IEEE 802.11n using open system (no security) and WPA2 security for Windows XP and Windows 7. Enabling WPA2 security results in approximately 4.4 Mbps less TCP throughput than open system for both IPv4 and IPv6 on Windows XP and up to 2.8 Mbps less TCP throughput for Windows 7. For both open system and WPA2 security, Windows 7 provides higher IPv4 and IPv6 bandwidth than Windows XP and IPv4 provides higher bandwidth than IPv6.

With the increased use of wireless local area networks (WLAN), performance evaluation of WLAN is becoming vital. The latest IEEE 802.11n WLAN provides benefits including greater reliability, better coverage area and higher throughput than 802.11g. The main advantage of the new 802.11n standard is higher throughput through spatial multiplexing, Multiple Input Multiple Output (MIMO) technology, that simultaneously sends and receives multiples streams of data. IEEE 802.11n standard is backward compatible with other IEEE 802.11 standards.

As IEEE 802.11n networks have become popular, any WiFi products released to the market are based on the $802.11 \mathrm{n}$ standard. Businesses looking to implement a wireless solution can greatly benefit from the 802.11n standard. However, the fact that wireless uses air as the medium for transmission raises critical questions about the security of WLAN. An appropriate encryption approach consequently must be used to provide confidentiality, integrity and authentication for the communicated traffic between different parties. The Wi-Fi Protected Access version 2 (WPA2) security encryption techniques is commonly used to address the wireless security problem. WPA2 is based on 802.1x and Advanced Encryption Standard (AES) which provides data encryption and user authentication, ${ }^{1}$ and it solves weaknesses in the prior cryptography methods such as Wired Equivalent Privacy (WEP) and WPA.

According to the registries that allocate network addresses around the world, the current Internet Protocol version 4 (IPv4) will run out of network addresses in the near future. ${ }^{2}$
Internet Engineering Task Force (IETF) therefore developed a new version of Internet Protocol, IPv6 that not only substantially expands the address space to $2^{128}$, but also has a raft of additional benefits that were lacking in IPv4, such as auto-configuration, more granular control of QoS (Quality of Service), better security features, and compatibility with the latest 3G mobile technology. New versions of popular enduser operating systems have capability for IPv6, and hardware vendors, software developers and Internet Service Providers (ISP) are moving towards offering support for IPv6. ${ }^{3}$

Windows 7 is the latest Windows operating system and is becoming popular but at the time of this research, Windows XP still had most of the market share. ${ }^{4}$

The main objective of this paper is to produce new results for bandwidth trade-off between open system and WPA2 security enabled Peer-to-Peer 802.11n WLAN for IPv6 and IPv4 using both Windows XP and Windows 7 operating systems. We established test-beds and analysed the effect of WAP2 security for IPv4 and IPv6 using TCP (Transmission Control Protocol) and UDP (User Datagram Protocol).

\section{Related Work}

Performance evaluation and comparison of IPv4 and IPv6 with and without security on different operating systems has been conducted by a number of researchers.

In 2004, Baghaei and colleagues ${ }^{3}$ carried out a study on the impact of security on 802.11 b networks using multiple clients. Their results demonstrated that adding encryption to an open system network results in decreasing the throughput by 
approximately $7 \%$ for WEP-64 and 10\% for WEP-128 using Windows XP.

In 2006, Ezedin and colleagues ${ }^{5}$ conducted a research on the impact of security on the performance of $802.11 \mathrm{~g}$ networks. They stated that the TCP throughput suffered a degradation of $4 \%$ on Windows XP and a $10 \%$ reduction in bandwidth on Windows Vista when WEP-64 was enabled.

In 2007, Filho and colleagues ${ }^{6}$ evaluated the impact of security mechanisms WEP and WPA on the performance of 802.11g network. Their results showed that when the security protocols such as WEP-64, WEP-128 and WPA were used in the IEEE 802.11g wireless network, the time of processing the traffic was increased and the throughput was decreased. The UDP throughput dropped by 4\% for WEP 64, 7\% for WEP 128, and 5\% for WPA on Windows XP.

In 2008, S.S. Kolahi and colleagues ${ }^{7}$ investigated the influence of wireless 802.11g LAN encryption methods on throughput for various Windows operating systems over a client-server network using Windows 2003 Server as the network operating system. Their results showed TCP traffic suffered a degradation of approximately 4-6\% with WPA for different Windows operating systems. UDP traffic degradations values were 1-2\% for WPA.

In 2009, S.S. Kolahi and colleagues ${ }^{8}$ conducted a study on the impact of overheads of security techniques for 802.11n on a client server wireless LAN using Windows XP, Windows Vista and Windows Server 2008 operating systems. Their results indicated that with WPA2 security enabled, Windows XP had approximately up to 8 Mbps less throughput than open systems, and Windows Vista had up to 11 Mbps less throughput than open system.

From the related work above, it is clear that the security techniques lower the available bandwidth and the amount of reduction depends on the wireless protocol, security mechanism, and the operating systems used. As there is a new operating system (Windows 7), and a relatively new wireless protocol (IEEE 802.11n) and, to the author's knowledge, there is no research to date in literature on studying WPA2 securitybandwidth tradeoff on Peer-to-Peer WLAN using Windows 7 and $802.11 n$, a new study is required. We obtain new results on the performance of 802.11n WLAN (with and without security) for both IPv4 and IPv6 protocols using Windows XP and Windows 7 operating systems. From the test-beds results, we study the WPA2 security-bandwidth trade-off.

\section{Experiment Setup}

To measure the performance of IPv4 and IPv6 on Windows XP and Windows 7, two client machines with identical hardware, Intel Core ${ }^{\mathrm{TM}} 2$ Duo1.87 GHz CPU, 2 GB RAM and Air Live Wn-5000 wireless PCI NIC, were connected. The access point used was Cisco Linksys WAP4410N 802.11n. We located the access point and two workstations well within two meters to maintain the optimum signal strength during the experiments. We did not install any additional third party software or vendor's updates to limit any extra parameters. The hardware test-bed setup remained constant for all experiments conducted.
The two operating systems setup and configuration are as follows:

- In test-bed I, Microsoft Windows XP Professional with SP3 (service pack 3) is installed on both client machines. Because Windows XP has enabled IPv4 only, IPv6 has to be explicitly installed and activated manually on the command line.

- In test-bed II, Microsoft Windows 7 Professional is installed on both client machines. By default both IPv4 and IPv6 are supported in Windows 7, they can be enabled and configured on the two workstations simultaneously by using the graphic interface.

Throughput (the number of bits transmitted per unit time) depends on several factors in a network, such as process limitations and hardware design. In order to eliminate the effect of such conditions, hardware with same characteristics is used in all of the tests to negate the effect of the processor limitations and hardware design.

Parameters used for the access point configuration are:

(a) Channel bandwidth - In addition to the direction of the transmission, a channel is characterized by its bandwidth. In general, the greater the bandwidth of the assigned channels, the higher the possible speed of transmission. The access point provided two options here, $20 \mathrm{MHz}$ and $40 \mathrm{MHz}$, and the latter was selected to utilize the full bandwidth.

(b) Guard Interval - Guard intervals are used to ensure that distinct transmissions do not interfere with one another. The purpose of the guard interval is to introduce immunity to propagation delays, echoes and reflections, to which digital data is normally very sensitive. This function was left appropriately to its default setting on the access point.

(c) CTS (Carpal Tunnel Syndrome) Protection Mode - This function boosts the access point's ability to detect all wireless connections but severely degrades performance, hence this setting was disabled to maximize performance.

(d) Beacon Interval - This function indicates the variable times in which clients meet the access point, this includes sending and receiving packets, and synchronism. ${ }^{6}$ This setting was best left at the default interval of $100 \mathrm{~ms}$.

(e) DTIM (Delivery Traffic Indication Message) Interval This setting specifies how often the access point broadcasts a Delivery Traffic Indication Message. According to the manual of the specific Linksys access point used in this project, lower settings ensure efficient networking. The default setting of $1 \mathrm{~ms}$ therefore was left for achieving the best results.

(f) RTS Threshold - RTS (Request-to-Send) is a signal sent from the transmitting station to the receiving station requesting permission to transmit data. This setting is used to decrease the problem of the hidden stations due to distance or signal blockage. ${ }^{9}$ The manual for the Linksys access-point recommended that this be left at the default setting of 2347 for optimum performance.

(g) Fragmentation Threshold - This specifies the number of bytes used to fragment the frames with a purpose to increase transfer reliability. If the frame size is very big, it can cause heavy interference and elevate the retransmissions rate. On the 
other hand, if the frame is too small, it will create overhead during the transmission and reduce the throughput rate. ${ }^{6,7}$ The parameter value for this was left at the default setting of 2346.

\section{Data Generation and Traffic Measurement Tool}

Netperf $2.4 .5^{10}$ was selected as the primary tool to analyze the performance of IPv4 and IPv6 on the two different operating systems over 802.11n WLAN. Netperf can be used to measure the performance of many different types of networks. It creates and sends TCP and UDP packets in either IPv4 or IPv6 networks, and provides tests for throughout. Netperf has also been used in the past for similar research such as studying the impact of wireless 802.11g security on performance of different Windows operating systems. ${ }^{5}$

As the focus is on security-bandwidth trade-off between open system and WPA2 security enabled, the metric used in the experiment is throughput (measured in Mbps). Bandwidth provides a valuable insight into network performance since it is the rate at which the data is transmitted over a network. Streams of packets are generated and sent from one computer to another for a period of about 60 seconds, and that usually generated one million packets of a particular packet size (one run). A total of 40 runs are carried out and the results averaged and standard deviation recorded to ensure accuracy of the results and to rule out any inconsistencies.

\section{Experimental Results}

Based on network of Figure 1, the TCP and UDP throughput were measured for both IPv4 and IPv6 on Windows 7 and Windows XP Peer-to-Peer network over 802.11n environment. The experiments were conducted to evaluate and compare the throughput of TCP and UDP on open system and WPA2 with different packet sizes such as 128, 384, 640, 896, 1152 and 1408 bytes.

Figure 1 shows TCP throughput results for Windows 7 with IPv4 and IPv6 protocols for open system and WPA2 security enabled using different packet sizes. From TCP throughput values, for all packet sizes, there were performance differences between IPv4 and IPv6. The variations in throughput were also different for the open system and WPA2 security enabled.

As can be seen from Figure 1, IPv4 with open system had the highest TCP throughput than the other scenarios. The maximum difference between IPv4 and IPv6 on open system was $1.88 \mathrm{Mbps}$ on packet size 128 bytes. On the same network with WPA2 security enabled, the highest point of difference between IPv4 and IPv6 can be noted at the packet size 1152 bytes where IPv4 provided 2.53 Mbps higher throughput than IPv6.

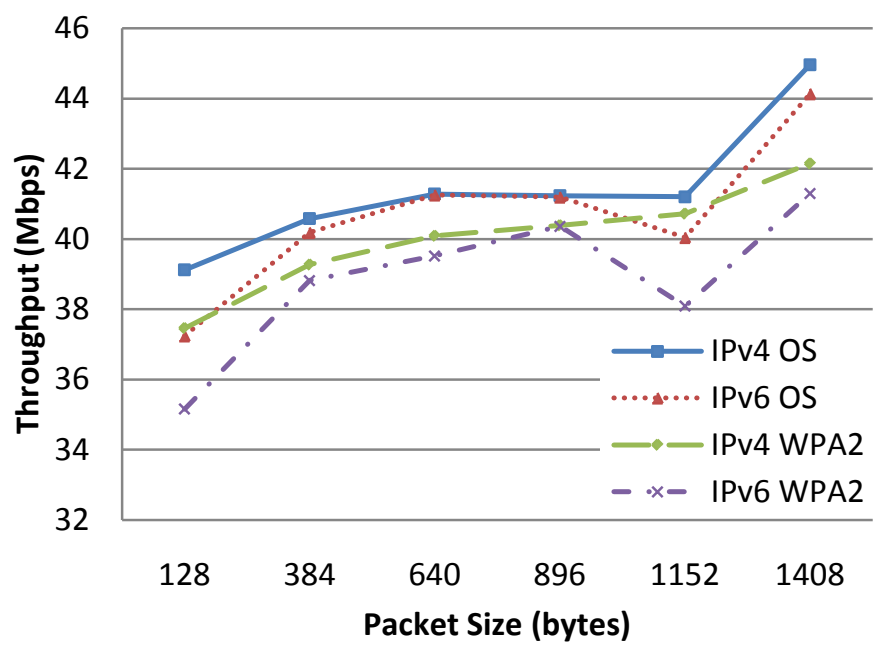

Figure 1: Windows 7 TCP Throughput for OS and WAP2

Analyzing the impact of WPA2 security on Windows 7, the throughput of both IPv4 and IPv6 is reduced when WPA2 security is enabled. The maximum gaps between open system and WPA2 were noticed at packet size 1408 bytes for both IPv4 and IPv6 where IPv4 provided 2.81 Mbps and IPv6 provided 2.83 Mbps higher throughput values in open system environment (Figure 1).

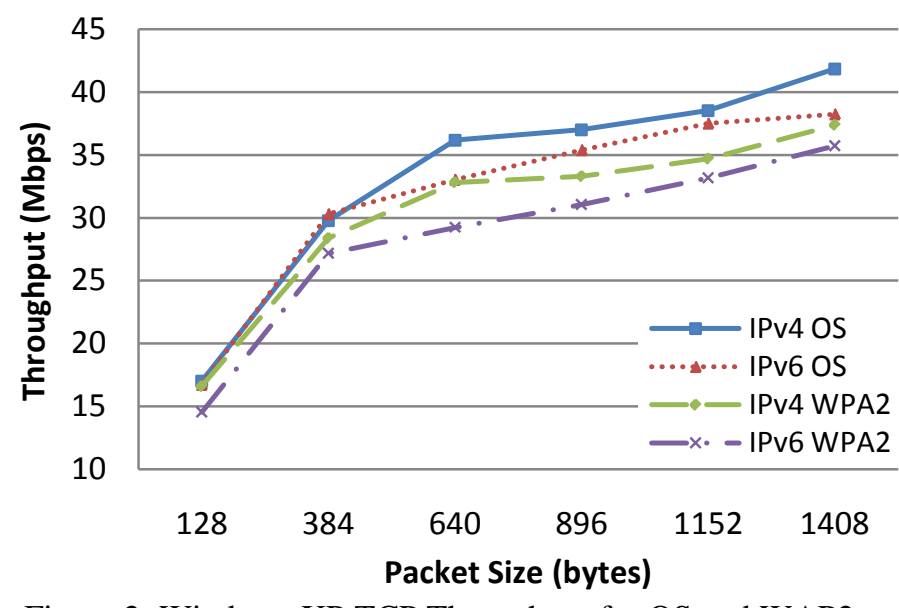

Figure 2: Windows XP TCP Throughput for OS and WAP2

Figure 2 shows the TCP throughput experimental results of Windows XP Peer-to-Peer wireless 802.11n network, and compares open system with WPA2 security for both IPv4 and IPv6. It can be seen that IPv4 performs better than IPv6 on both open system and WAP2. The highest point of difference between IPv4 and IPv6 on open system was at packet size of 1408 bytes where IPv4 outperformed IPv6 by $3.6 \mathrm{Mbps}$. With WPA2 security enabled, the difference was highest at the packet size of 640 bytes where IPv4 had 3.56 Mbps higher throughput than IPv6.

Figure 2 also shows the security-bandwidth trade-off in windows XP environment. The highest point of difference between open system and WPA2 on IPv4 was at packet size of 1408 bytes where open system outperformed WPA2 by 4.46 
Mbps. For IPv6, the maximum difference in throughput between open system and WPA2 was at packet sizes 896 and 1152 bytes where open system outperformed WPA2 by 4.33 Mbps for both packet sizes.

Comparing Windows XP and Windows 7 performances (Figures 1 and 2), Windows 7 had a higher TCP throughput than Windows XP for both IPv4 and IPv6 on open system. The most noticeable difference between Windows XP and Windows 7 performance was noticed at packet size of 128 bytes for both IPv4 and IPv6, where IPv4 on Windows 7 had 22 Mbps more TCP throughput, and IPv6 on Windows 7 has 20 Mbps higher TCP throughput. Also on the WPA2 security enabled, Windows 7 again had a better TCP throughput than Windows XP for both IPv4 and IPv6. The highest point of differences between Windows XP and Windows 7 were at packet size of 128 bytes for both IPv4 and IPv6, where both IPv4 and IPv6 on Windows 7 had 21 Mbps more TCP throughput.

The standard deviation for the TCP throughput results are recorded in the following table:

TABLE 1

STANDARD DEVIATION FOR TCP THROUGHPUT

\begin{tabular}{|c|c|c|c|c|c|c|c|c|}
\hline \multirow{2}{*}{$\begin{array}{c}\text { Packet } \\
\text { size } \\
\text { (Bytes) }\end{array}$} & \multicolumn{4}{|c|}{ Windows 7 } & \multicolumn{4}{c|}{ Windows XP } \\
\cline { 2 - 9 } & Open System & \multicolumn{2}{|c|}{ WPA2 } & \multicolumn{2}{c|}{ Open System } & \multicolumn{2}{c|}{ WPA2 } \\
\hline \multirow{2}{*}{128} & IPv4 & IPv6 & IPv4 & IPv6 & IPv4 & IPv6 & IPv4 & IPv6 \\
\cline { 2 - 9 } & 0.95 & 0.83 & 0.99 & 0.89 & 0.55 & 0.98 & 0.31 & 0.71 \\
\hline 384 & 1.00 & 1.13 & 0.97 & 0.88 & 0.98 & 0.99 & 0.89 & 0.98 \\
\hline 640 & 0.99 & 1.07 & 0.94 & 0.90 & 0.87 & 0.88 & 0.73 & 0.90 \\
\hline 896 & 0.75 & 1.05 & 0.99 & 0.85 & 0.93 & 0.96 & 0.88 & 0.99 \\
\hline 1152 & 0.86 & 0.96 & 0.96 & 0.90 & 0.92 & 0.99 & 0.93 & 0.97 \\
\hline 1408 & 0.97 & 0.99 & 0.95 & 0.58 & 0.90 & 0.98 & 0.92 & 0.73 \\
\hline
\end{tabular}

UDP results obtained from the test-bed for the two operating systems with IPv4 and IPv6 protocols are presented in Figures 3 and 4.

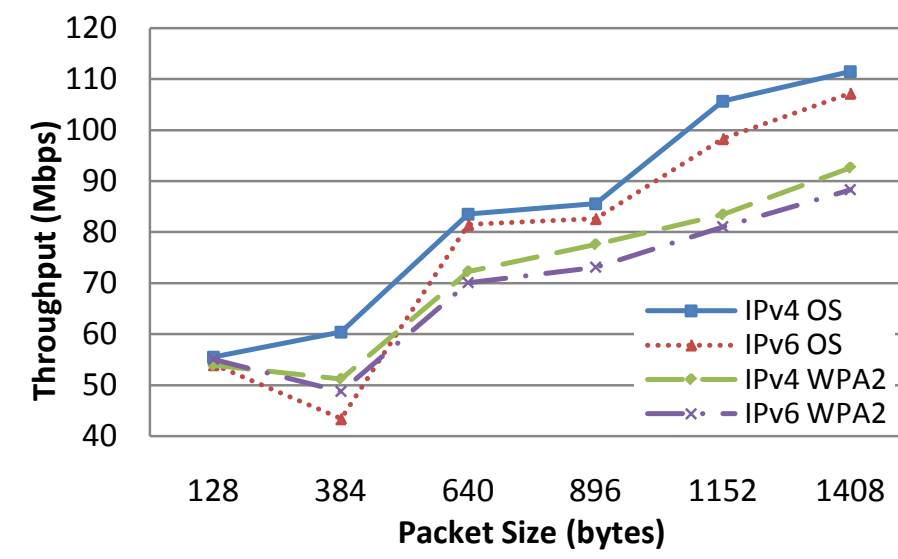

Figure 3: Windows 7 UDP Throughput for OS and WAP2
Figure 3 shows the UDP throughput results for IPv4 and IPv6 on the Windows 7 running on open system and WPA2 security enabled. IPv4 with open system had the highest throughput than other scenarios. The highest point of difference between IPv4 and IPv6 running on open system can be noted at the packet size of 384 bytes where IPv4 provided 16.95 Mbps higher throughput than IPv6. With the WPA2 security enabled, IPv4 also provided higher throughput than IPv6 for all the packet sizes, and the maximum difference between IPv4 and IPv6 was 4.46 Mbps on packet size of 896 bytes.

We note that UDP performs better on open system than WPA2 for both IPv6 and IPv4. The highest gap between the open system and the WPA2 enabled was noticed at the packet size of 1152 bytes for IPv4 and 1408 bytes for IPv6 where IPv4 with no security provided 22.23 Mbps higher bandwidth than WPA2, and IPv6 with no security provided higher throughput of 18.91 Mbps compared to WPA2 environment.

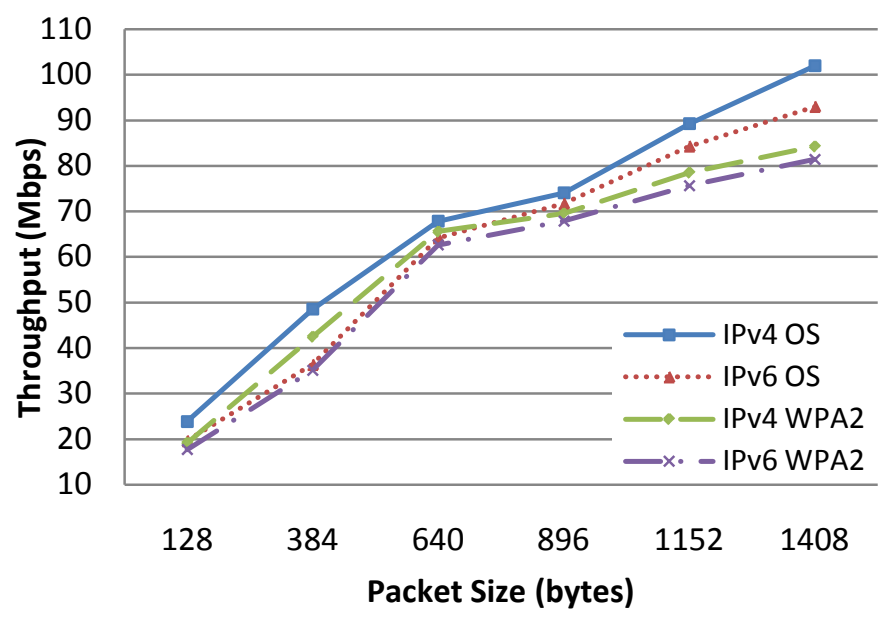

Figure 4: Windows XP UDP Throughput for OS and WAP2

Figure 4 shows the UDP throughput experiment results of Windows XP Peer-to-Peer wireless 802.11n network. It can be seen that IPv4 performs better than IPv6 on both open system and WAP2. On open system, IPv4 had higher throughput than IPv6 on all packet sizes. The maximum difference in throughput between IPv4 and IPv6 was at packet size of 384 bytes where IPv4 outperformed IPv6 by $12.14 \mathrm{Mbps}$. Also on WPA2, IPv4 had better throughput than IPv6 on all packet sizes. The maximum difference in UDP throughput between IPv4 and IPv6 on WPA2 was once again at packet size of 384 bytes where IPv4 outperformed IPv6 by 7.35 Mbps.

Using Windows XP with IPv4 and IPv6, open system again outperformed WPA2. The highest gap in UDP throughput between open system and WPA2 on IPv4 was at the packet size of 1408 bytes where open system had 17.68 Mbps more throughput than WPA2. For IPv6, the maximum difference in UDP throughput between open system and WPA2 was at packet size of 1408 bytes where open system outperformed WPA2 by 11.56 Mbps. 
As can be seen from Figures 3 and 4, comparing the two operating systems, on open system, Windows 7 had a higher UDP throughput than Windows XP for both IPv4 and IPv6 for most packet sizes. The difference is most noticeable at 128 bytes for both IPv4 and IPv6, where Windows 7 had 31.69 Mbps higher throughput for IPv4 and 34.37 Mbps more throughput for IPv6 than Windows XP. Also with WPA2 security enabled, Windows 7 outperformed Windows XP on all packet sizes for both IPv4 and IPv6. The maximum difference between Windows 7 and Windows XP was at packet size of 128 bytes for both IPv4 and IPv6, where IPv4 on Windows 7 had 34.77 Mbps more UDP throughput, and IPv6 on Windows 7 had 37.37 Mbps higher UDP throughput.

The standard deviation for the above throughput results are recorded in the following table:

TABLE 2

STANDARD DEVIATION FOR UDP THROUGHPUT

\begin{tabular}{|c|c|c|c|c|c|c|c|c|}
\hline \multirow{2}{*}{$\begin{array}{c}\text { Packet } \\
\text { size } \\
\text { (Bytes) }\end{array}$} & \multicolumn{4}{|c|}{ Windows 7 } & \multicolumn{4}{c|}{ Windows XP } \\
\cline { 2 - 9 } & Open System & \multicolumn{2}{|c|}{ WPA2 } & \multicolumn{2}{c|}{ Open System } & \multicolumn{2}{c|}{ WPA2 } \\
\hline \multirow{2}{*}{128} & IPv4 & IPv6 & IPv4 & IPv6 & IPv4 & IPv6 & IPv4 & IPv6 \\
\cline { 2 - 9 } & 0.93 & 0.91 & 0.44 & 0.40 & 0.98 & 0.86 & 0.71 & 0.98 \\
\hline 384 & 0.61 & 0.88 & 0.96 & 0.95 & 0.99 & 0.96 & 0.98 & 0.96 \\
\hline 640 & 0.97 & 0.88 & 0.96 & 0.94 & 0.88 & 0.97 & 0.90 & 0.98 \\
\hline 896 & 0.82 & 1.00 & 0.95 & 1.08 & 0.96 & 0.96 & 0.99 & 0.97 \\
\hline 1152 & 0.88 & 1.28 & 0.98 & 0.95 & 0.99 & 0.91 & 0.97 & 0.95 \\
\hline 1408 & 0.96 & 1.07 & 0.90 & 0.98 & 0.98 & 0.98 & 0.73 & 0.90 \\
\hline
\end{tabular}

The UDP throughputs are higher than the TCP on both open system and WPA2 security enabled. This is due to UDP being a connectionless protocol and does not use any form of error correction and therefore does not send any acknowledgements. The source does not have to wait to receive any acknowledgments.

The above results are for a Peer-to-Peer wireless LAN when both links to the access points are wireless. The TCP and UDP throughput are much less than what we achieved (120 Mbps for TCP and 175 Mbps for UDP) in IEEE 802.11n wireless client-server networks experiments where one of the links (to the server) was cable. Due to its CSMA/CA media access control that needs processing time for RTS (request to send) and CTS (clear to send), having two wireless links can lower the bandwidth compared to the WLAN client-server network.

The lower throughput gained on IPv6 than in IPv4 is due to the drawback of having a larger overhead in IPv6 (IPv6 has a 40-bit header while IPv4 has a 20-bit header) over IPv4. ${ }^{11}$ This increases in header size had major implications for the performance of the newer IPv6, resulting in lower bandwidth.

The gain in TCP and UDP throughput values as packet size increases is likely due to the amortization of overheads associated with larger user packet sizes. ${ }^{13}$.
The lower throughput results obtained when WPA2 security is enabled (compared to no security) is due to the security overhead of WPA2 (16 bytes/packet) that increases the processing time per packet for security, and negatively impacts on the overall throughput performance. ${ }^{12}$

\section{Conclusion}

The choice of operating system has an impact on WLAN bandwidth. Windows 7 provided higher WLAN throughput than Windows XP on both open system and WPA2 security enabled. Results also showed that, due to higher overhead, IPv6 provided lower bandwidth than IPv4. We determined the difference in IPv4 and IPv6 throughputs for TCP and UDP.

There was a bandwidth-security trade off when WPA2 security was enabled for both IPv4 and IPv6 using Windows XP or Windows 7. Enabling WPA2 resulted in approximately up to $4.4 \mathrm{Mbps}$ less TCP throughput than open system for both IPv4 and IPv6 on Windows XP, and up to $2.8 \mathrm{Mbps}$ less TCP throughput than open system for both IPv4 and IPv6 on Windows 7. With WPA2 security enabled, IPv4 had approximately up to $18 \mathrm{Mbps}$ less UDP throughput and IPv6 had up to 12 Mbps less UDP throughput on Windows XP than the system with no security. On Windows 7, with WPA2 security enabled, IPv4 and IPv6 had up to 20 Mbps lower UDP throughput than open system.

\section{Future Work}

In future, we plan to extend this study by incorporating more operating systems and more metrics. In addition, the performance comparison of Windows and Linux Systems with IPv4 and IPv6 using both open systems and WPA2 security on 64-bit operating system will be investigated.

\section{Acknowledgment}

The authors would like to thank UNITEC Institute of Technology, Mustafa Safdari, and Mulugeta Argawe for their assistance during this project.

\section{References}

1. A. D. Potorac and D. Balan. "The Impact of Security Overheads on 802.11 WLAN Throughput," Computer Science and Control Systems, vol. 2, 2009, pp. 47-52.

2. OECD report, Internet Address Space: Economic Considerations in the Management of IPv4 and in the Deployment of IPv6, OECD Ministerial Meeting, Seoul, Korea, 17-18 June 2008.

3. N. Baghaei and R. Hunt, "IEEE 802.11 Wireless LAN Security Performance Using Multiple Clients," in Proceedings of the 12th IEEE International Conference on Networks, Vol. 1, Nov. 2004, pp. 299-303.

4. W3Schools.com, "OS Platform Statistics," August 2010; www.w3schools.com/browsers/browsers_os.asp

5. B. Ezedin, B. Mohammed, A. Amal, S. Hanadi Al, K. Huda, and M. Meera Al, "Impact of Security on the Performance of Wireless-Local Area Networks,” Innovations in Information Technology, 2006, pp. 1-5.

6. E.J.M.A. Filho, P.N.L. Fonseca, M.J.S. Leitao, and P.S.F. de Barros, "Security versus Bandwidth: The Support of Mechanisms WEP e WPA in 802.11g Network," IFIP International Conference on Wireless and Optical Communications Networks, WOCN '07, pp. 1-5, 2007. 
7. S.S. Kolahi, S. Narayan, D.T. Du, Y. Sunarto, P. Mani, "The Impact of Wireless LAN Security on Performance of Different Windows Operating Systems,” IEEE Symposium on Computers and Communications, 2008, pp. 260-264.

8. S. S. Kolahi, Z. Qu, B. K. Soorty, and N. Chand,“The Impact of Security on the Performance of IPv4 and IPv6 Using 802.11n Wireless LAN," New Technologies, Mobility and Security (NTMS), 2009 3rd International Conference on, 2009, pp. 1-4.

9. D. Akin, and J. Geier, “802.11 PHY layers,” CWAP - certified wireless analysis professional official study guide, Mc.Graw-Hill, 2004, pp. 353355.

10. R. Jones, “Netperf 2.4.5,” Netperf.org, 30 March 2010; www.netperf.org/netperf/NetperfNew.html
11. R. Murugesan, S. Ramadass, R. Budiarto, "Improving the Performance of IPv6 Packet Transmission over LAN," 2009 IEEE Symposium on Industrial Electronics and Applications (ISIEA 2009), October, 2009, pp. 182-187.

12. Barka Ezedin, Boulmalf Mohammed, "On The Impact of Security on the Performance of WLANs,” Journal of Communication, vol. 2, NO. 4, Jun 2007.

13. S. Zeadally, and L. Raicu, "Evaluating IPv6 on Windows and Solaris," Internet Computing, IEEE, vol. 7, no. 3, 2003, pp. 51-57. 